TRANSACTIONS OF THE

AMERICAN MATHEMATICAL SOCIETY

Volume 358, Number 1, Pages 373-390

S 0002-9947(05)03670-6

Article electronically published on March 31, 2005

\title{
POLYGONAL INVARIANT CURVES FOR A PLANAR PIECEWISE ISOMETRY
}

\author{
PETER ASHWIN AND AREK GOETZ
}

\begin{abstract}
We investigate a remarkable new planar piecewise isometry whose generating map is a permutation of four cones. For this system we prove the coexistence of an infinite number of periodic components and an uncountable number of transitive components. The union of all periodic components is an invariant pentagon with unequal sides. Transitive components are invariant curves on which the dynamics are conjugate to a transitive interval exchange. The restriction of the map to the invariant pentagonal region is the first known piecewise isometric system for which there exist an infinite number of periodic components but the only aperiodic points are on the boundary of the region. The proofs are based on exact calculations in a rational cyclotomic field. We use the system to shed some light on a conjecture that PWIs can possess transitive invariant curves that are not smooth.
\end{abstract}

\section{INTRODUCTION}

Piecewise isometries (PWIs) are simple families of dynamical systems that show a lot of dynamical complexity while not being hyperbolic in even the weakest of senses; classical examples in one dimension are the rotation on the circle or, more generally, interval exchange transformations (IETs) (see, e.g. [17]). PWIs have also been found to arise in several applications such as in simple digital filter models [18, 2], billiard systems 21] and other area-preserving maps [20, 19].

Two-dimensional (planar) PWIs are considerably more mysterious than IETs, even when one is limited to polygonal domains of continuity (atoms) and cases that are invertible; in contrast to the case for IETs, periodic points are common even when all rotations are by irrational amounts. It is conjectured that PWIs typically have an infinite number of periodic points and that the symbolic dynamics has polynomial complexity. The former conjecture has been verified only in a special case [8] whereas the latter only verified in a different special case [1, 14.

Moreover for such PWIs a variety of $\omega$-limit sets are possible; these include finite sets (periodic points), unions of circles (invariant circles) and two-dimensional sets, such as one can trivially find for piecewise isometries that correspond to a product of

Received by the editors November 22, 2002 and, in revised form, March 22, 2004.

2000 Mathematics Subject Classification. Primary 37B10, 37E15; Secondary 11R11, 20C20, $68 \mathrm{~W} 30$.

Key words and phrases. Piecewise isometry, interval exchange transformation, cells.

The work on this article commenced during the second author's visit to Exeter sponsored by the LMS. The second author was partially supported by NSF research grant DMS 0103882, and the San Francisco State Presidential Research Leave. We thank Michael Boshernitzan for interesting conversations and helpful suggestions. Symbolic computations were aided by Mathematica routines, some of which were developed in connection with a project by Goetz and Poggiaspalla. 
two incommensurate irrational rotations on a square unit cell. There is numerical evidence that other invariant sets are also possible, under the proviso that the symbolic dynamics are aperiodic. In particular 2] found numerical examples of what appear to be invariant curves that are continuously embedded circles but such that the embedding is not differentiable at any point.

In this paper we examine an example of PWI $T: \mathbb{C} \rightarrow \mathbb{C}$, illustrated in Figure 1 where there is a non-smooth invariant curve for $T$. The map has a pentagonal region $Q$ bounded by a pentagon with unequal sides such that $T(Q)=Q$. We show that within this pentagon there are an infinite number of periodic points such that the only accumulation points of this set are on the boundary of $Q$.

As such, the map restricted to $Q$ has a set of periodic points of unbounded period, but in contrast to previous examples [1], in this case the set of aperiodic points is $\partial Q$, and it has dimension one (and hence the conjecture in Remark 4, p. 217 of [4] is false). Such behavior is also in contrast to the case for one-dimensional interval exchange transformations, where there is a uniform bound on the period if all points are periodic [6].

The dynamics on the boundary of $Q$ is such that a first return map is a rotation with golden mean $\gamma=\frac{1+\sqrt{5}}{2}$ rotation number. Outside of $Q$ there is a continuum of invariant curves on which first return maps can be found that are also golden mean rotations.

The structure of the paper is as follows; for clarity we have separated the results and the proofs, which are based on finite computations with integer arithmetic. In the remainder of this section we fix the notation and define the map we consider. We summarize the nature of tools used in the proof in Section 3.1. The proofs of results are included afterwards and we conclude the article with various remarks and numerical observations. These numerics suggest that the polygonal invariant curves exist for other rational rotation maps and that they may become 'non-smooth invariant curves' for maps including irrational rotations.

In this article we will ignore a countable union of lines on which the orbits are not well defined; namely those points that hit the boundary of an atom at some point in the future. This set, usually referred to as the exceptional set [8, 9] has Hausdorff dimension one. Its closure, which includes all points that have aperiodically coded trajectories, may have Hausdorff dimension up to two. Two-dimensional, rationally coded cells (sets of points that follow the same eventually periodic coding pattern) have interiors free of exceptional sets and boundaries contained within the exceptional set and the union of the boundaries of the atoms.

Interactive multimedia located at http://math.sfsu.edu/goetz/Research/ illustrate definitions and selected results from this article.

1.1. Definition of the generating map $T: \mathbb{C} \rightarrow \mathbb{C}$. Let $\rho=e^{\pi i / 5}$ be the primitive tenth root of unity. Partition the plane $C$ into four atoms, one half plane and three cones as illustrated in Figure 1 and defined as follows.

$$
\begin{aligned}
& P_{0}=\left\{r \rho^{s} \mid r>0, s \in(0,5)\right\}, \\
& P_{1}=\left\{r \rho^{s} \mid r>0, s \in(5,8)\right\}, \\
& P_{2}=\left\{r \rho^{s} \mid r>0, s \in(8,9)\right\}, \\
& P_{3}=\left\{r \rho^{s} \mid r>0, s \in(9,10)\right\} .
\end{aligned}
$$



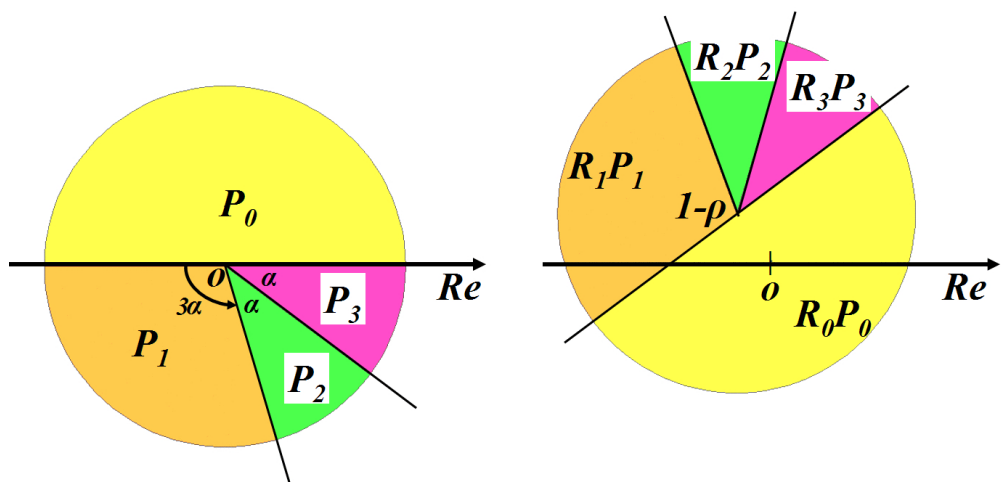

Figure 1. The action of the generating piecewise rotation $T$. The map $T$ permutes four cones, $P_{0}, P_{1}, P_{2}$, and $P_{3}$ and then translates them by $1-\rho$ as shown.

We define the rotations

$$
\begin{aligned}
& R_{0}(z)=\rho^{6} z-1+\rho, \\
& R_{1}(z)=\rho^{8} z-1+\rho, \\
& R_{2}(z)=\rho^{4} z-1+\rho, \\
& R_{3}(z)=\rho^{2} z-1+\rho
\end{aligned}
$$

and the piecewise rotation $T: \mathbb{C} \rightarrow \mathbb{C}$ is defined as $T z=R_{j} z$ if $z \in P_{j}$. One can generalize this map to allow for irrational rotations $\rho$; see [5].

\section{Results}

2.1. The existence of a polygon fixed by $T$. In this section we demonstrate that there exists a centrally located pentagon $Q$ that is invariant under $T$.

By $\left[a_{1}, \ldots, a_{n}\right]$ we denote a closed polygon with vertices $a_{k} \in \mathbb{C}$; in the case $\left[a_{1}, a_{2}\right]$ it is simply the line segment joining $a_{1}$ and $a_{2}$. Given any subset $A \subset \mathbb{C}$ we define (first) return time $k_{A}(z)$ to be the least integer $k \geq 1$ such that $T^{k}(z) \in A$. For any $z \in A$ we define

$$
T_{A}(z)=T^{k_{A}(z)}(z)
$$

to be the (first) return map to $A$ and the transient part of the orbit of return to $A$ to be

$$
\operatorname{tran}_{T}^{A}(z)=\left\{z, \ldots, T^{k_{A}(z)-1}(z)\right\} .
$$

We define

$$
a=1, b=\rho+\rho^{3}, c=-1+\rho+\rho^{3}, d=-1, e=-\rho^{3}, g=\rho-\rho^{3}
$$

(see Figure 2).

Proposition 2.1 (Invariance of the pentagon $Q$ ). The pentagon $Q=[b, c, d, e, g]$ is fixed by $T$, namely $T(Q)=Q$.

The dynamics outside and inside of $Q$ are characterized later on in Theorems 2.1 and 2.4. 


\subsection{Existence and dynamics of invariant polygonal curves outside the} pentagon $Q$. The dynamics outside the pentagon $Q$ are dramatically different than inside $Q$. The complement of $Q$ splits into invariant polygonal curves on which there are periodic and minimal components. Let

$$
\begin{array}{lll}
b_{0}=b+\delta \rho & \text { and } & b_{1}=b+\delta \rho^{2}, \\
c_{0}=c+\delta \rho^{3} & \text { and } & c_{1}=c+\delta \rho^{4}, \\
d_{0}=d+\delta \rho^{5} & \text { and } & d_{1}=d+\delta \rho^{6}, \\
e_{0}=e+\delta \rho^{7} & \text { and } & e_{1}=e+\delta \rho^{8}, \\
g_{0}=g+\delta \rho^{9} & \text { and } & g_{1}=g+\delta .
\end{array}
$$

Fix $\delta \geq 0$. Let $\mathcal{C}_{\delta}$ be the closed curve (illustrated in Figure 2) defined as

$$
\begin{gathered}
\mathcal{C}_{\delta}=\left[b_{0}, b_{1}\right] \cup\left[b_{1}, c_{0}\right] \cup\left[c_{0}, c_{1}\right] \cup\left[c_{1}, d_{0}\right] \cup\left[d_{0}, d_{1}\right] \\
\cup\left[d_{1}, e_{0}\right] \cup\left[e_{0}, e_{1}\right] \cup\left[e_{1}, g_{0}\right] \cup\left[g_{0}, g_{1}\right] \cup\left[g_{1}, b_{0}\right] .
\end{gathered}
$$

Note that for $\delta=0$ this is simply the boundary of the pentagon $Q$.
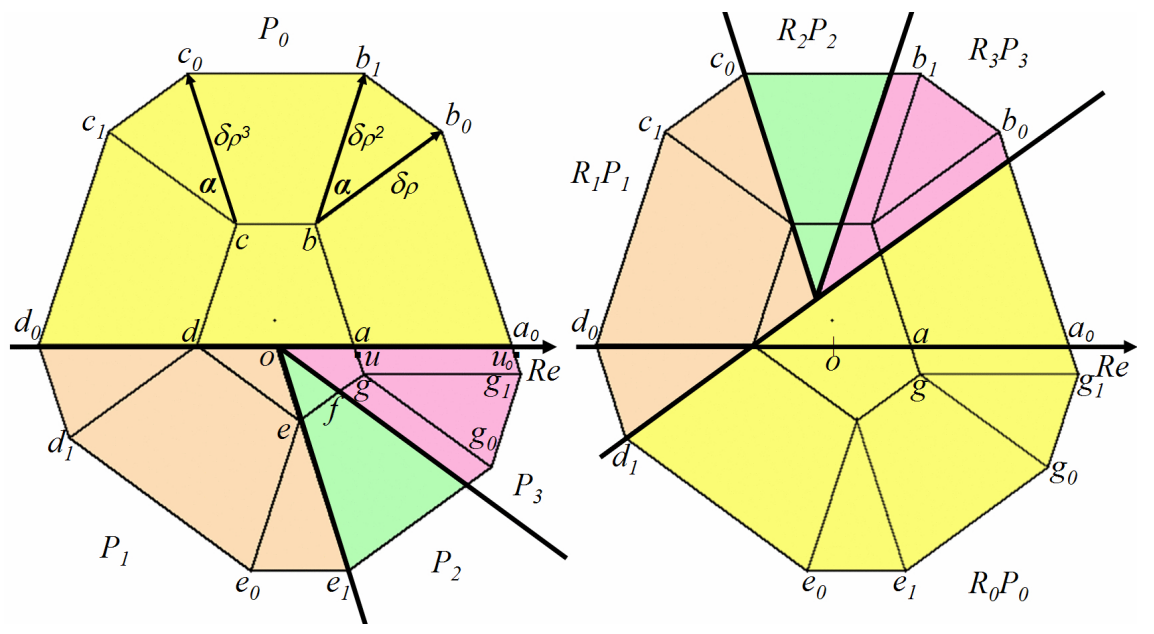

Figure 2. The invariant pentagon $Q=[a, b, c, d, e, g]$ and an invariant 10 -sided curve $\mathcal{C}_{\delta}$ outside $Q$. The points $u$ and $u_{0}$ are as in Theorem 2.1.

The following theorem states the existence of invariant curves $\mathcal{C}_{\delta}$. It also describes the dynamics on these curves in detail. We show that $T$ acts on $\mathcal{C}_{\delta}$ as an interval exchange transformation that splits into two components, one periodic and one uniquely ergodic. The dynamics on the uniquely ergodic component reduces to the irrational rotation by the golden mean for a suitable return map.

Theorem 2.1 (Existence and dynamics on the invariant curves). (i) Existence. The curves $\mathcal{C}_{\delta}(\delta \geq 0)$ together with the pentagon $Q$ exhaust the plane, that is,

$$
\mathbb{C}=Q \cup \bigcup_{\delta \in \mathbb{R}^{+}} \mathcal{C}_{\delta} .
$$

Moreover, each curve, $\mathcal{C}_{\delta}$ is $T$-invariant,

$$
T\left(\mathcal{C}_{\delta}\right)=\mathcal{C}_{\delta} .
$$


(ii) The periodic component. The map $\left.T\right|_{\mathcal{C}_{\delta}}$ is a continuous permutation of the five intervals:

$$
\left[b_{0}, b_{1}\right] \stackrel{T}{\longrightarrow}\left[e_{0}, e_{1}\right] \stackrel{T}{\longrightarrow}\left[d_{0}, d_{1}\right] \stackrel{T}{\longrightarrow}\left[c_{0}, c_{1}\right] \stackrel{T}{\longrightarrow}\left[g_{0}, g_{1}\right] \stackrel{T}{\longrightarrow}\left[b_{0}, b_{1}\right] .
$$

(iii) The uniquely ergodic component. Let $a_{0}=a+\delta$. The interval $\left[g_{1}, a_{0}\right] \subset \mathcal{C}_{\delta}$. The orbit of $\left[g_{1}, a_{0}\right]$ is disjoint to the periodic orbit $\left[b_{0}, b_{1}\right]$. Together, these two orbits cover the curve $\mathcal{C}_{\delta}$, that is,

$$
\mathcal{C}_{\delta}=\bigcup_{0 \leq n \leq 4} T^{n}\left[b_{0}, b_{1}\right] \cup \bigcup_{n \geq 0} T^{n}\left[g_{1}, a_{0}\right] .
$$

(iv) Golden mean return map. The first return map $T_{\left[g_{1}, a_{0}\right]}$, is independent of $\delta$ and is an exchange of two intervals $\left[g_{1}, u_{0}\right]$ and $\left[u_{0}, a_{0}\right]$, where $g_{1}=\delta+\rho-\rho^{3}$, $a_{0}=\delta+1, u_{0}=\delta-2+3 \rho-2 \rho^{3} \in\left[g_{1}, a_{0}\right]$ and

$$
T_{\left[g_{1}, a_{0}\right]}(z)= \begin{cases}z+\left(a_{0}-u_{0}\right) & \text { if } z \in\left[g_{1}, u_{0}\right] \\ z+\left(g_{1}-u_{0}\right) & \text { if } z \in\left[u_{0}, a_{0}\right] .\end{cases}
$$

The ratio

is the golden mean.

$$
\frac{u_{0}-g_{1}}{a_{0}-u_{0}}=\frac{1+\sqrt{5}}{2}
$$

Remark 2.2. Since by Theorem 2.1 the action on $\partial Q=C_{0}$ is minimal, it follows that the closure of a periodic cell in $Q$ must be disjoint from $\partial Q$, a conclusion of Corollary 2.1.

Remark 2.3. By permutation (2.8) in Theorem 2.1] entire cone sectors, $\left\{\left[b_{0}, b_{1}\right] \mid \delta \geq\right.$ $0\},\left\{\left[e_{0}, e_{1}\right] \mid \delta \geq 0\right\},\left\{\left[d_{0}, d_{1}\right] \mid \delta \geq 0\right\},\left\{\left[c_{0}, c_{1}\right] \mid \delta \geq 0\right\},\left\{\left[g_{0}, g_{1}\right] \mid \delta \geq 0\right\}$, are permuted by rotations. Hence in order to obtain invariant curves $\mathcal{C}_{\delta}$ one can replace the straight segments in these sectors with any other curve contained within the sectors with matching endpoints.

2.3. The dynamics inside the pentagon $Q$. In the previous section we remarked that $T(Q)=Q$. In this section we summarize the dynamics inside $Q$. We show that the pentagon $Q$ consists of an infinite number of cells, all of which are periodic. We illustrate the distribution of periods of the cells in the pentagon.

Given a point $z \in \mathbb{C}$, let $\operatorname{per}_{T}(z)$ denote the period of $z$ under $T$. (If $z$ is not periodic we let $\operatorname{per}_{T}(z)=\infty$.) We denote the Hausdorff distance between two sets $A, B$ by $\operatorname{dist}(A, B)$.

Theorem 2.4 (Distribution of periodic points within the pentagon). (i) Periodicity. Every point in $\operatorname{int}(Q)$ is periodic.

(ii) Distribution of periodic cells. Fix an arbitrary distance $\epsilon>0$. For all $z \in \operatorname{int}(Q)$,

$$
\sup \left\{\operatorname{per}_{T}(z) \mid \operatorname{dist}(z, \partial Q) \geq \epsilon\right\}<\infty .
$$

Moreover,

$$
\liminf _{\epsilon \rightarrow 0^{+}}\left\{\operatorname{per}_{T}(z) \mid \operatorname{dist}(z, \partial Q)<\epsilon\right\}=\infty .
$$

Corollary 2.1. All periodic cells in the pentagon $Q$ are a positive distance away from the boundary of $Q$. 
Corollary 2.2. For every subset $U \subset Q$ that is a positive distance away from the boundary of $Q$, there exists $n>0$ such that $T^{n}$ is the identity on $U$.

Finally, we remark that map $K=\left.T\right|_{\operatorname{int}(Q)}$ is the first known example of an invertible piecewise rotation with convex atoms for which there are periodic points with unbounded period but no aperiodic points except on the boundary.

Corollary 2.3. Let $K=\left.T\right|_{\text {int }(Q)}$. The map $K$ is an invertible piecewise rotation with four convex polygonal atoms. Let $E$ be the exceptional set for the piecewise rotation $K$. Then (i) all points under $K$ are periodic, however, the set of periods is unbounded. Moreover, (ii) $\bar{E}=E$.

Corollary 2.3 describes a new phenomenon that is not present in dimension one. Every interval exchange transformation splits into a finite number of transitive and periodic components [6]. In particular, an interval exchange transformation for which there are only periodic components must have a uniform upper bound on the period.

\section{Proofs}

3.1. Symbolic computations. In this section we outline a rigorous verification method of the computations included in the proofs. Symbolic software (Mathemat$i c a$ and Maple) was used to aid in the computations. Similar techniques are used in [11] and computer aided symbolic computations for real number field extensions are used in [15, 16].

Let $\rho=e^{\pi i / 5}$. Let $\mathbb{Q}[\rho]$ denote a collection of polynomial expressions in $\rho$ with rational coefficients. (Actually, $\mathbb{Q}[\rho]$ is a field obtained by adjoining either $\rho$ or $\rho^{2}$.

In the proofs below we frequently use verifications of the following type. Given a point $z \in \mathbb{Q}[\rho]$ and a polygon $W$, we need to decide whether $z \in W$. This reduces to the verification if $z \in H$, where $H$ is the closed half-plane containing the center of mass of $W, o$, determined by the line passing through $a$ and $b$, two (consecutive) vertices of $W$. Then $z \in H$ if the real number

$$
x=((o-a) \overline{(a-b)}-\overline{(o-a)}(a-b))((z-a) \overline{(a-b)}-\overline{(z-a)}(a-b)) \geq 0 .
$$

The verification of the last inequality is immediate and exact since each real number in $\mathbb{Q}[\rho]$ is of the form $m+n \sqrt{5}$ for some rationals $m, n$.

\subsection{Proof of Proposition 2.1.}

Proof. The proof is an elementary verification that the polygons $R_{0}\left(P_{0} \cap Q\right), R_{1}\left(P_{1} \cap\right.$ $Q), R_{1}\left(P_{2} \cap Q\right)$ and $R_{2}\left(P_{3} \cap Q\right)$ do not overlap and that they are contained in $Q$.

\subsection{Proof of Theorem 2.1.}

3.3.1. Proof of (i), (ii). Examining Figure 2, we can verify that the intervals that compose $\mathcal{C}_{\delta}$ are mapped in a one-to-one manner onto each other. In particular, we verify that the intervals $\left[b_{0}, b_{1}\right],\left[e_{0}, e_{1}\right],\left[d_{0}, d_{1}\right],\left[c_{0}, c_{1}\right]$ and $\left[g_{0}, g_{1}\right]$ are cyclically permuted in this order (2.8), meaning that the fifth iterate on these intervals acts as the identity. This is an elementary calculation whose flavor we now illustrate. For example we show that $T\left[b_{0}, b_{1}\right]=\left[e_{0}, e_{1}\right]$. Since $b_{0}, b_{1} \in P_{0}$, it is enough to trace the endpoints. For example,

$$
R_{0} b_{0}=\rho^{6}(b+\delta \rho)-1+\rho=\rho^{6}\left(\rho+\rho^{3}+\delta \rho\right)-1+\rho=-\rho^{3}+\delta \rho^{7}=e_{0} .
$$



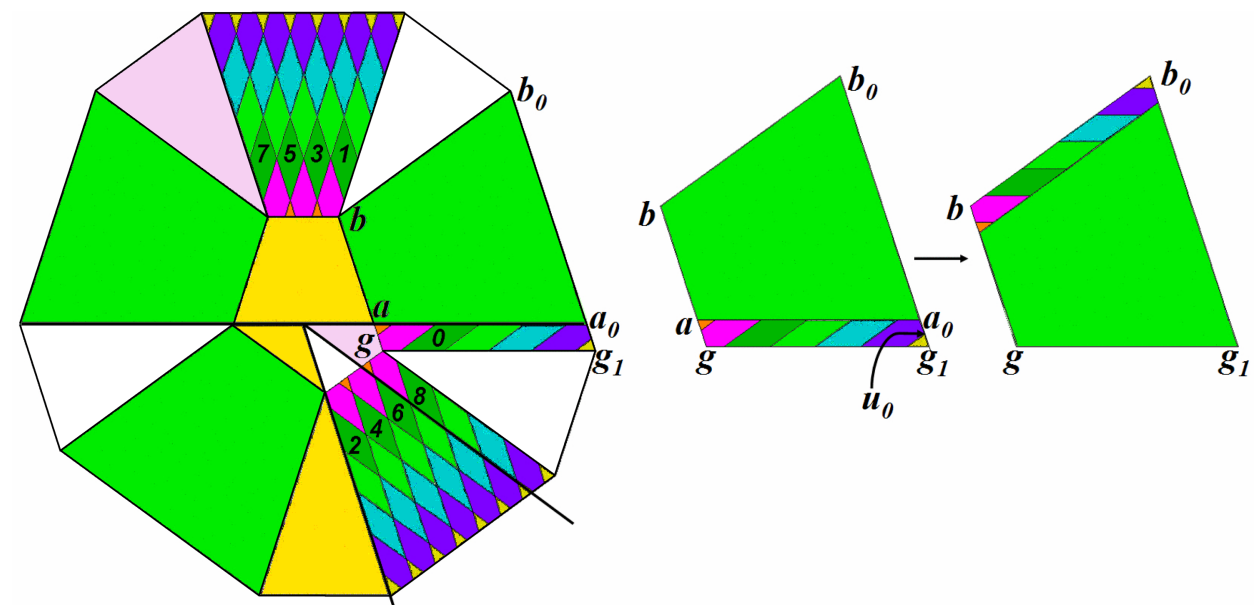

FiguRE 3. Illustration of the return map to the wedge $\left[g, g_{1}, b_{0}, b\right]$. In the right figure we illustrated the transient orbit of the wedge in the original system $T: \mathbb{C} \rightarrow \mathbb{C}$. The regions that return to the wedge after the same number of iterates are shaded in the same way.

In the above calculation the simplification uses the fact that the cyclotomic $C_{10}(z)=$ $1-z+z^{2}-z^{3}+z^{4}$ vanishes at $z=\rho$.

Similarly, one can verify that the union of all the other intervals on the curve are mapped to each other in a one-to-one manner, although this is not a simple permutation. This gives (2.7).

Any point outside $Q$ lies on such a curve for some $\delta>0$ and so the complement of $Q$ is exhausted by the curves $\mathcal{C}_{\delta}$, (2.6) and concludes the proof of (i) and (ii).

3.3.2. Proof of (iii) and (iv). We divide the required derivation of the return maps into two stages. Stage 1 is the following lemma.

Lemma 3.1. The return map to the segment $\left[g_{1}, b_{0}\right]$ (Figure 3) is the exchange of intervals as follows:

$$
T_{\left[g_{1}, b_{0}\right]}(z)= \begin{cases}z+\left(g_{1}-a_{0}\right) & \text { if } z \in\left[a_{0}, b_{0}\right], \\ z+k\left(a_{0}-g_{1}\right)+\rho^{3}, & \text { if } z \in\left[g_{1}, a_{0}\right] .\end{cases}
$$

In the second line of (3.1), $k$ is the integer such that $z+k\left(a_{0}-g_{1}\right)+\rho^{3} \in\left[g_{1}+b_{0}-\right.$ $\left.a_{0}, b_{0}\right]$. Moreover, the T-orbit of any point in $\left[g_{1}, b_{0}\right]$ (disregarding the exceptional set) is $\mathcal{C}_{\delta}-\bigcup_{0 \leq n \leq 4} T^{n}\left[b_{0}, b_{1}\right]$.

Note that one can easily verify that the intersection of the exceptional set with any $C_{\delta}$ is a countable union of points and hence zero dimensional.

Remark 3.2. The map $T_{\left[g_{1}, b_{0}\right]}$ is an exchange of three intervals for almost all choices of $\delta$. See Figure 3 , 
Proof. First, we observe that

$$
\begin{aligned}
& {\left[a_{0}, b_{0}\right] \stackrel{T}{\longrightarrow}\left[e_{0}, d_{1}\right] \stackrel{T}{\longrightarrow}\left[d_{0}, c_{1}\right] \stackrel{T}{\longrightarrow}\left[g_{1}, g_{1}+b_{0}-a_{0}\right]} \\
& \begin{array}{cccc}
\cap & \cap & \cap & \cap \\
P_{0} & P_{1} & P_{0} & {\left[g_{1}, b_{0}\right]}
\end{array}
\end{aligned}
$$

The interval $\left[a_{0}, b_{0}\right] \subset\left[g_{1}, b_{0}\right]$ returns to $\left[g_{1}, b_{0}\right]$ translated by $g_{1}-a_{0}$. This concludes the first part of the verification of the upper equation of (3.1).

On the other hand, consider $z \in\left[g_{1}, a_{0}\right]$; this is mapped into atom $P_{0}$ and then alternates between $P_{2}$ and $P_{0}$, being successively shifted along the intervals $\left[e_{1}, g_{0}\right]$ and $\left[b_{1}, c_{0}\right]$ until it enters $P_{3}$ and is then mapped back onto the interval $\left[b_{0}+a_{0}-g_{1}, b_{0}\right]$.

Note that the length of $\left[e_{1}, g_{0}\right]$ is $\gamma+\gamma \delta$, that of $\left[g_{1}, a_{0}\right]$ is $1-\gamma$ and that of $\left[g_{1}, b_{0}\right]$ is $2+\gamma \delta$, where $\gamma=\frac{\sqrt{5}-1}{2}=0.61803398$.

Hence on the first return to $\left[g_{1}, b_{0}\right], z$ will be shifted by a distance being the difference in lengths of $\left[e_{1}, g_{0}\right]$ and $\left[g_{1}, b_{0}\right]$ modulo adding on a multiple of the length of $\left[g_{1}, a_{0}\right]$. Hence on first return $z$ is translated by a distance

$$
2+\gamma \delta-1-\gamma \delta+k(1-\gamma)=1+k(1-\gamma)
$$

relative to $g_{1}$ in the direction $\rho^{3}$, for some $k>0$ integer such that the image lies within $\left[b_{0}-a_{0}+g_{1}, b_{0}\right]$. This means that the map has the form

$$
z \mapsto z+k\left(a_{0}-g_{1}\right)+\rho^{3}
$$

for some $k$ (the number of iterates to return on this interval will take two possible values).

We now use Lemma 3.1 to investigate the return of $T_{\left[g_{1}, b_{0}\right]}$ to $\left[g_{1}, a_{0}\right] \subset\left[g_{1}, b_{0}\right]$. (This is the return map $T_{\left[g_{1}, a_{0}\right]}$ of $T$ to $\left[g_{1}, a_{0}\right]$.)

Lemma 3.3. The return map $T_{\left[g_{1}, b_{0}\right]}$ induces a return map on $\left[g_{1}, a_{0}\right]$ given by Theorem 2.1(iv).

Proof. This can be obtained by noting that the map on $\left[g_{1}, a_{0}\right]$ must have the form

$$
T_{\left[g_{1}, a_{0}\right]}(z)=z+l\left(a_{0}-g_{1}\right)+\rho^{3}=z+l\left(1-\rho+\rho^{3}\right)+\rho^{3}
$$

for some integer $l$ such that $T(z) \in\left[g_{1}, a_{0}\right]$. One can verify that independent of $\delta$, $l=2$ or $l=3$ and

$$
T_{\left[g_{1}, a_{0}\right]}(z)= \begin{cases}z+3-3 \rho+2 \rho^{3} & \text { if } z \in\left[g_{1}, u_{0}\right] \\ z+2-2 \rho+\rho^{3} & \text { if } z \in\left[u_{0}, a_{0}\right]\end{cases}
$$

which corresponds to the stated map, where $u_{0}=-2+3 \rho-2 \rho^{3}+\delta$.

We now prove (2.9). Observe that the transient orbit of $\left[g_{1}, a_{0}\right]$ under $T_{\left[g_{1}, b_{0}\right]}$ is $\left[g_{1}, b_{0}\right]$. Since the transient $T$-orbit of $\left[g_{1}, b_{0}\right]$ is $\mathcal{C}_{\delta}-\bigcup_{0 \leq n \leq 4} T^{n}\left[b_{0}, b_{1}\right]$ (Lemma 3.1 ), we conclude that the transient $T$-orbit of $\left[g_{1}, a_{0}\right]$ is also $\overline{\mathcal{C}}_{\delta}-\bigcup_{0 \leq n \leq 4} T^{n}\left[b_{0}, b_{1}\right]$, giving (2.9). 

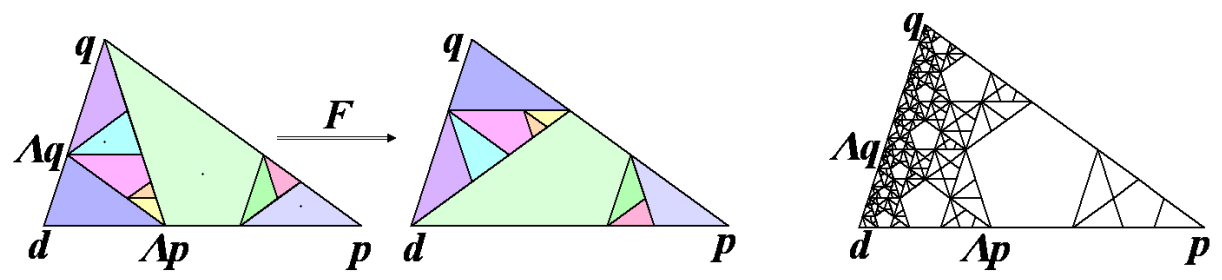

Figure 4 . The action of the return map $F=T_{\triangle_{1}}$ and the partition of $\triangle_{1}$ into periodic cells.

3.4. Proof of Theorem 2.4. In order to prove Theorem 2.4, we show that the pentagon $Q$ splits into a finite number of periodic families of cells, as well as the orbit of a small triangle near the vertex $d, \triangle_{1}=[d, p, q]$ (Figure [6). Let

$$
F(z)=T_{\triangle_{1}}(z)
$$

be the return map to $\triangle_{1}$ (Figure 4 ).

The central goal is to prove an analogous result to Theorem 2.4 for the map $F: \triangle_{1} \rightarrow \triangle_{1}$ which, as we will see later (Lemma 3.11), contains essentially all dynamics of the map $T$.

\subsection{Dynamics of the map $F$.}

Lemma 3.4 (Key Lemma). Let $F$ be the return map $T_{\triangle_{1}}$ to $\triangle_{1}=[d, p, q]$, where

$$
d=-1, p=1-3 \rho^{2}+3 \rho^{3} \text { and } q=-1+5 \rho-8 \rho^{2}+5 \rho^{3} .
$$

Fix an arbitrary distance $\epsilon>0$. Then $F$ is well defined and for all $z \in \operatorname{int}\left(\triangle_{1}\right)$ and all $\epsilon>0$,

$$
\sup \left\{\operatorname{per}_{F}(z) \mid \operatorname{dist}(z,[d, q]) \geq \epsilon\right\}<\infty .
$$

Moreover,

$$
\liminf _{\epsilon \rightarrow 0^{+}}\left\{\operatorname{per}_{F}(z) \mid \operatorname{dist}(z,[d, p])<\epsilon\right\}=\infty .
$$

The proof of Lemma 3.4 involves several steps. The first is to show that $F$ is conjugate to the return of $F$ on one of its atoms. This enables us to show that all points in $\triangle_{1}$ are $F$-periodic. We then investigate the distribution of cells in $\triangle_{1}$.

We begin by computing the return map to $\triangle_{1}$. Let $\lambda=2-\rho-\rho^{-1}=0.38196601$, let

$$
\Lambda(z)=\lambda(z+1)-1
$$

be the contraction about $d=-1$ with contraction ratio $\lambda$ and let

$$
\triangle_{2}=\Lambda \triangle_{1}
$$

be the scaled version of $\triangle_{1}$ (see Figure 4).

Lemma 3.5 (Self-similarity of $F$ ). Let $F_{\triangle_{2}}$ be the F-return map of $\triangle_{2}$ to $\triangle_{1}$. Then $F_{\triangle_{2}}$ is conjugate to $F$ under the similarity $\Lambda$.

$$
F_{\triangle_{2}}(z)=\Lambda^{-1} F \Lambda(z) \text { for all } z \in \triangle_{1} .
$$




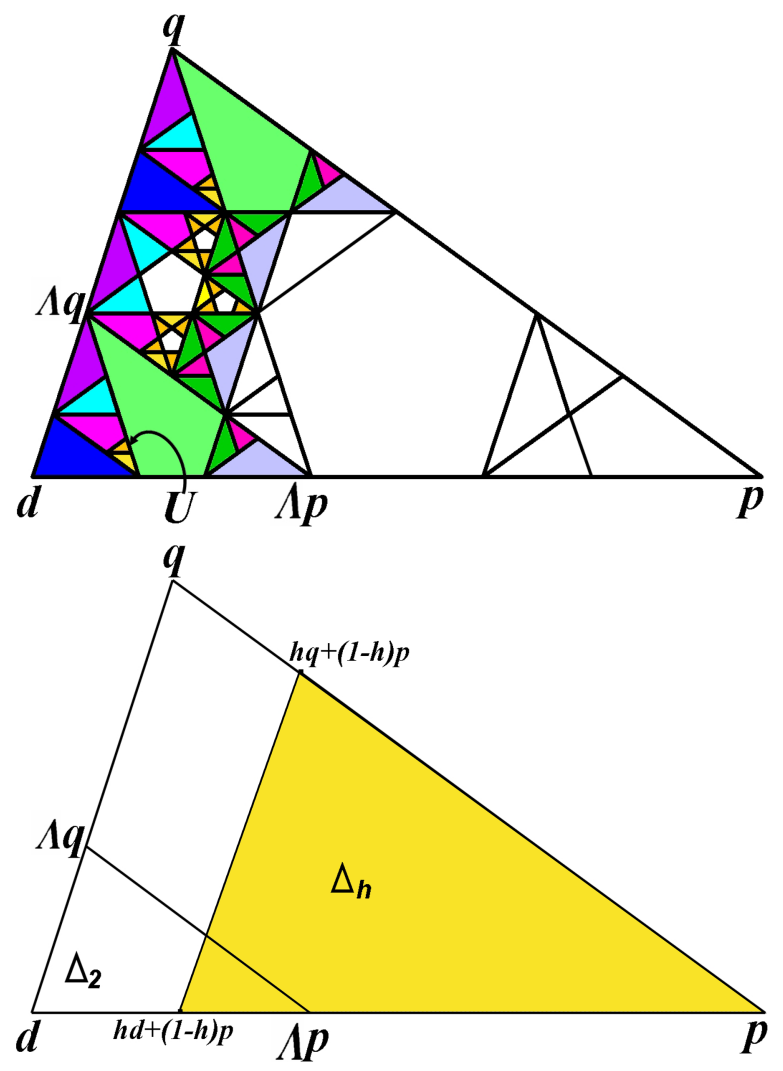

Figure 5. The upper figure illustrates the transient orbit of the induced atoms of $F_{\triangle_{2}}\left(\triangle_{2}=[d, \Lambda p, \Lambda q]\right)$ shown as shaded polygons within $\triangle_{1}$. The white polygons are periodic cells that never enter $\triangle_{2}$. For details, see Lemma 3.6. The right figure is an illustration to Lemma 3.9

The total area of the orbits of the polygons listed above is $A_{p}=50962-$ $101924 \rho+\frac{329833 \rho^{2}}{4}-\frac{77863 \rho^{3}}{4}$. The area of the orbit of $\triangle_{2}$ is $A_{r}=-\left(\frac{101941}{2}\right)+$ $101941 \rho-82472 \rho^{2}+19469 \rho^{3}$. We compute the area $A_{r}$ by adding up the areas of the 10 induced atoms of $\mathcal{P}_{F}$ (Lemma 3.5) together with all their $F$-iterates that are disjoint from $\triangle_{2}$.

Together these areas add up to the area of $\triangle_{1}, A_{\triangle_{1}}=A_{r}+A_{p}=-\left(\frac{17}{2}\right)+17 \rho-$ $\frac{55 \rho^{2}}{4}+\frac{13 \rho^{3}}{4}$ which completes the proof of Lemma 3.6 .

We now prove two geometric lemmas which will be used in proving Lemma 3.4, The first describes the "quasi equidistant" behavior of transient orbits $\operatorname{tran}_{F}^{\triangle_{2}} \triangle_{2}$ near the boundary $[d, q]$ of $\triangle_{1}$.

Let $U=\left[-77+89 \rho-21 \rho^{2}-34 \rho^{3}, 67-55 \rho-21 \rho^{2}+55 \rho^{3},-22+89 \rho-110 \rho^{2}+\right.$ $55 \rho^{3}$ ]. In Figure 5, left, $U$ is the smallest shaded (in orange) triangle contained in $\triangle_{2}=[d, p, q]\left(U\right.$ is an atom of $\left.T_{\triangle_{2}}\right)$. 
Lemma 3.7. The triangle $U$ is a periodic cell. Moreover, for the map $F: \triangle_{1} \rightarrow \triangle_{1}$ defined in Table 1 and all $z \in \triangle_{2}-U$,

$$
\frac{\operatorname{dist}\left(\operatorname{tran}_{F}^{\triangle_{2}}(z),[d, q]\right)}{\mu_{1}} \leq \operatorname{dist}(z,[d, q]) \leq \frac{\operatorname{dist}\left(\operatorname{tran}_{F}^{\triangle_{2}}(z),[d, q]\right)}{\mu_{2}},
$$

where $\mu_{1}=1+\rho^{2}-\rho^{3}=\frac{1+\sqrt{5}}{2}$, and $\mu_{2}=\rho^{2}-\rho^{3}=\frac{-1+\sqrt{5}}{2}$.

Remark 3.8. The exclusion of $z \in U$ from the estimate (3.8) is necessary to obtain an inequality sharp enough for an argument in Lemma 3.9. We will need $\mu_{1} \lambda<1$. If points $z \in U$ are included in the estimate, then $\mu_{1}$ would be $1 / \lambda$.

Proof. Since $\operatorname{tran}_{F}^{\triangle_{2}}$ consists of the atoms of $F_{\triangle_{2}}$ together with their finite iterates, and since each of these iterates is convex, we have verified inequality (3.8) for all the vertices of the iterates of the atoms of $F_{\triangle_{2}}$.

For any $h \in(0,1)$ we define the triangle

$$
\triangle_{h}=h\left(\triangle_{1}-p\right)+p
$$

scaled by a factor $h$ with vertex $p$ fixed (see Figure 5).

Lemma 3.9. For any $h \in(1,0)$ we have the containment

$$
\triangle_{1-\mu_{2}+\mu_{2} h} \subset \operatorname{tran}_{F}^{\triangle_{2}}\left(\Lambda \triangle_{h}\right) \cup \triangle_{h} \cup M,
$$

where $M$ is a finite union of periodic cells and the transient F-orbit of the periodic cell $U$.

Proof. The proof is an immediate geometric observation, the application of the first part of inequality (3.8) in the previous lemma, and the algebraic simplification $1-(1-h) \lambda \mu_{1}=1-\mu_{2}+\mu_{2} h$.

In Figure 5 the set $M$ is represented by non-shaded polygons and the transient orbit of the small periodic triangular cell $U$ by the shaded cells.

Lemma 3.10 (Distribution of periodic cells in $\left.\triangle_{1}\right)$. For all $h \in(0,1), \operatorname{per}_{F}\left(\triangle_{h}\right)$ is a finite set.

Proof. Since the function $\operatorname{per}_{F}: \triangle_{1} \rightarrow[1, \infty]$ is invariant under iteration,

$$
\operatorname{per}_{F}\left(\operatorname{tran}_{F}^{\triangle_{2}}\left(\Lambda \triangle_{h}\right)\right)=\operatorname{per}_{F}\left(\Lambda \triangle_{h}\right) \text {. }
$$

From Lemma 3.9, equation (3.9), we thus obtain

$$
\operatorname{per}_{F}\left(\triangle_{1-\mu_{2}+\mu_{2} h}\right) \subset \operatorname{per}_{F}\left(\Lambda \triangle_{h}\right) \cup \operatorname{per}_{F}\left(\triangle_{h}\right) \cup \operatorname{per}(M) .
$$

Suppose that for some $h, \operatorname{per}_{F}\left(\triangle_{h}\right)$ is finite. Observe that the right-hand side of (3.10) is also finite. The set $\operatorname{per}_{F}(M)$ is finite since $M$ is a finite union of periodic cells. By conjugation of $F: \triangle_{1} \rightarrow \triangle_{1}$ to its return map to $\Lambda \triangle_{1}$ (Lemma 3.5), set $\operatorname{per}_{F}\left(\Lambda \triangle_{h}\right)$ is finite because $\operatorname{per}_{F}\left(\triangle_{h}\right)$ is finite.

By (3.10), we conclude that $\operatorname{per}_{F}\left(\triangle_{1-\mu_{2}+\mu_{2} h}\right)$ is also finite. Since $\operatorname{per}_{F}\left(\triangle_{0}\right)$ is finite, it follows that for all $h_{n}$ defined inductively $h_{n}=1-\mu_{2}+\mu_{2} h_{n-1} ; h_{0}=0$, $\operatorname{per}_{F}\left(\triangle_{h}\right)$ is finite. Since $h_{n} \rightarrow 1$ (the global attractor for the function $h \mapsto$ $1-\mu_{2}+\mu_{2} h$ is $h=1$ ), this yields the desired conclusion of Lemma 3.10 . 
Finally, to conclude the proof of key Lemma 3.4, we need to show inequality (3.4). (Lemma 3.10 implies inequality (3.3)). Suppose, on the contrary, that

$$
\liminf _{\epsilon \rightarrow 0^{+}}\left\{\operatorname{per}_{F}(z) \mid \operatorname{dist}(z,[d, p])<\epsilon\right\}=M<\infty .
$$

Then there is a $\left\{n_{k}\right\} \subset \triangle_{1}, n_{k} \rightarrow[d, q]$ such that $\operatorname{per}_{F}\left(n_{k}\right)=M$.

Since $\triangle_{1}$ splits into a disjoint union of periodic cells located at a positive distance from $[d, q]$ and the transient orbit of $\triangle_{2}, \operatorname{tran}_{F}^{\triangle_{2}} \triangle_{2}$ (Lemma 3.6), we may assume that $\left\{n_{k}\right\} \subset \operatorname{tran}_{F}^{\triangle_{2}} \triangle_{2}$. This means that there is a sequence

$$
\left\{m_{k}\right\} \subset \triangle_{2}
$$

such that $T^{l} m_{k}=n_{k}(l \geq 0)$.

Since the return time to $\triangle_{2}$ under $F$ is at least 2 for all points in $\triangle_{2}\left(F\left(\triangle_{2}\right)\right.$ and $\triangle_{2}$ are disjoint and a positive distance apart), we have

$$
2 \operatorname{per}_{T_{\triangle_{2}}}\left(m_{k}\right) \leq \operatorname{per}_{F}\left(n_{k}\right)=M .
$$

Moreover since $n_{k} \rightarrow[d, q]$, by Lemma 3.7 (the second part of the inequality (3.8)), we obtain

$$
m_{k} \rightarrow[d, q] \text { as } k \rightarrow \infty .
$$

From (3.12), (3.13), (3.14), we obtain

$$
\liminf _{\epsilon \rightarrow 0^{+}}\left\{\operatorname{per}_{T_{\triangle_{2}}}(z) \mid \operatorname{dist}(z,[d, p])<\epsilon\right\} \leq M / 2 .
$$

On the other hand, since the maps $F$ and $T_{\triangle_{2}}$ are conjugate (Lemma 3.5), the left-hand side of (3.11) and the left-hand side of (3.15) must be equal, which is a contradiction. This concludes the proof of (2.12), and also the proof of the key Lemma 3.4

3.5.1. Proof of Theorem 2.4(i). We now return to the dynamics on the pentagon $Q$.

Lemma 3.11 (Decomposition of the pentagon). The pentagon int $(Q)$ splits into a disjoint union of the transient orbit of $\triangle_{1}$ and a finite number of periodic cells. All these periodic cells are located at a positive distance from the boundary $\partial Q$. (Figure 6).

Proof. The proof is a rigorous computer-aided verification in the field $\mathbb{Q}(\rho)$. The 19 polygons are listed in Table 3.

We verify that each polygon in the list above is periodic, its orbit is a positive distance away from $\partial Q$, and it follows a unique coding, different for each polygon. The total area of the transient orbits of these polygons is $A_{p}=517200-1034400 \rho+$ $\frac{3347395 \rho^{2}}{4}-\frac{790205 \rho^{3}}{4}$. There are (Lemma 3.5) 10 distinct induced atoms on $\triangle_{1}$ whose total transient area is $A_{r}=-\left(\frac{1034401}{2}\right)+1034401 \rho-\frac{3347391 \rho^{2}}{4}+\frac{790213 \rho^{3}}{4}$. Together these areas add up to the area of $Q, A_{Q}=A_{r}+A_{p}=-\left(\frac{1}{2}\right)+\rho+\rho^{2}+2 \rho^{3}$. This concludes the proof of Lemma 3.11 .

Note that Lemma 3.4 implies that every point in $\overline{\triangle_{1}}-[d, q]$ is periodic; Theorem 2.4(i) then immediately follows from Lemma 3.11. 


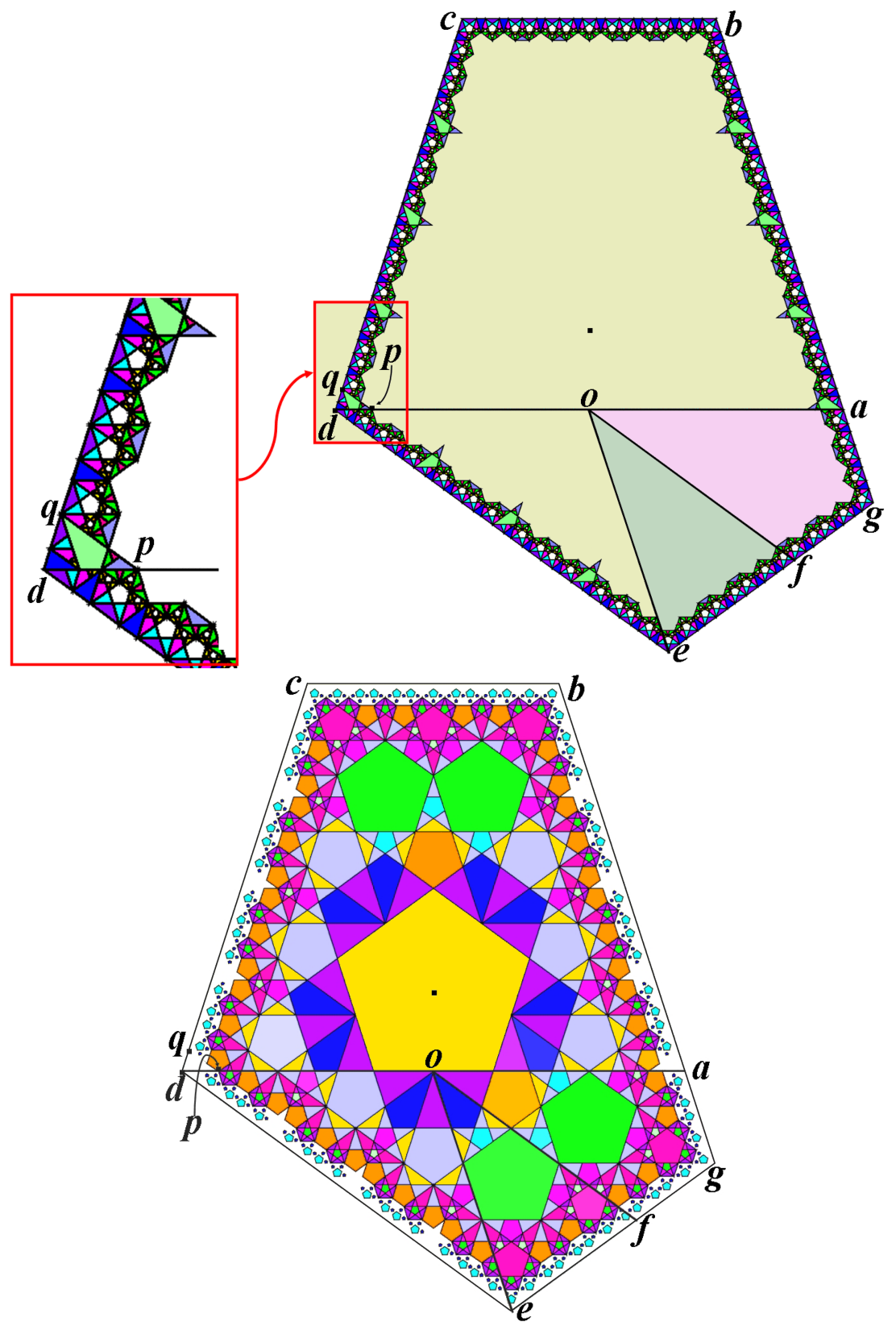

Figure 6 . The upper figure illustrates the band around the edge of $Q$ that is the $T$-orbit of $\triangle_{1}=[d, p, q]$ in $Q$. The lower figure illustrates the complement of the orbit of $\triangle_{1}$. This complement splits into 19 families of periodic polygonal cells listed in Table 3. For details, refer to Lemma 3.11. 
TABLE 3. The list of the vertices of 19 periodic polygonal cells. A vertex $v=a_{0}+a_{1} \rho+a_{2} \rho^{2}+a_{3} \rho^{3} \in \mathbb{Q}(\rho)$ is represented by $\left(a_{0}, a_{1}, a_{2}, a_{3}\right)$. The union of the orbits of these cells together with the orbit of $\triangle_{1}$ is the pentagon $Q$.

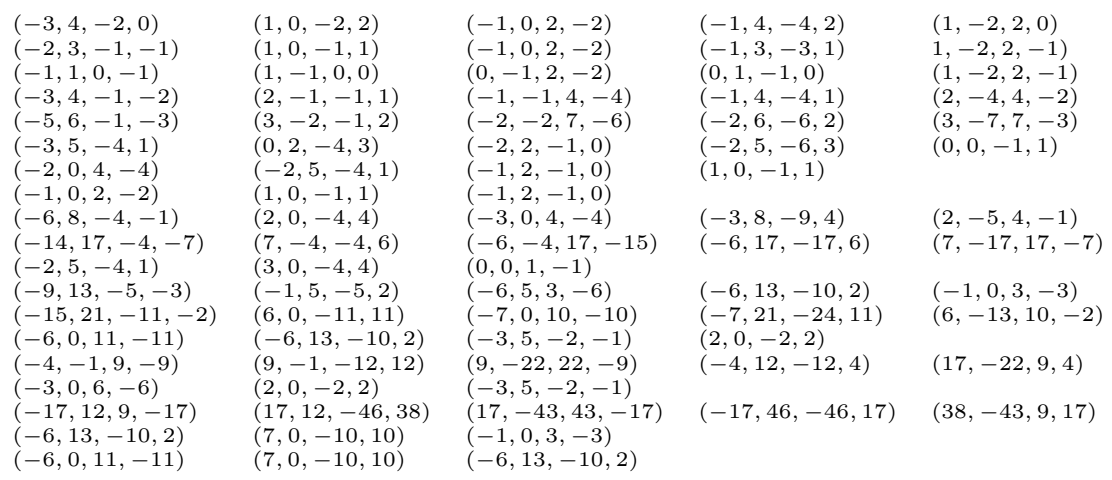

3.5.2. Proof of Theorem 2.4(ii). We now show that the distribution of periodic cells on $\triangle_{1}$ extends to the pentagon $Q$.

Lemma 3.12. There are positive constants $\eta_{1}$, and $\eta_{2}$ such that for all $z \in \triangle_{1}$ with $\operatorname{dist}(z,[d, q])<h$ we have

$$
\frac{\operatorname{dist}\left(\operatorname{tran}_{T}^{\triangle_{1}}(z),[d, q]\right)}{\eta_{1}} \leq \operatorname{dist}(z, \partial Q) \leq \frac{\operatorname{dist}\left(\operatorname{tran}_{F}^{\triangle_{1}}(z), \partial Q\right)}{\eta_{2}} .
$$

Remark 3.13. Unlike in Lemma 3.7, the values of the constants are not needed for the remainder of the proof.

Proof. There are five atoms $\left\{U_{1}, U_{2}, U_{3}, U_{4}, U_{5}\right\}$ of $F$ that have boundaries that intersect the invariant line $[d, q]$ (Figure 5). All remaining five atoms of $F$ have transient $T$-orbits in $Q$ located at a positive distance to $\partial Q$. It suffices to prove Lemma 3.12 for $z \in\left\{U_{1}, U_{2}, U_{3}, U_{4}, U_{5}\right\}$. Since each of the cells is convex, it is enough to check Lemma 3.12 for each vertex $v$ of each atom $\left\{U_{1}, U_{2}, U_{3}, U_{4}, U_{5}\right\}$, that (a) if $v \in[d, q]$, then the whole transient orbit of $v$ is in $\partial Q$, (b) if $v \notin[d, q]$, then no transient iterate of $v$ is in $\partial Q$. This is done with the help of symbolic software. (We iterate the orbit of a vertex $v$ of an atom $U_{i}$ by following the coding of the atom $U_{i}$.)

Fix an arbitrary $\epsilon>0$. We now show that the set $\operatorname{per}_{T}(\{z \in Q, \operatorname{dist}(z, \partial Q) \geq \epsilon\})$ is finite. By Lemma 3.12 (by the first part of inequality (3.16) ) and by Lemma 3.11 .

$$
\{z \in Q, \operatorname{dist}(z, \partial Q) \geq \epsilon\} \subset \operatorname{tran}_{T}^{\triangle_{1}}\left(\triangle_{\epsilon \eta_{1}}\right) \cup M,
$$

where $M$ is a union of a finite number of periodic cells.

Observe that by invariance of $\operatorname{per}_{T}$ under iteration,

$$
\operatorname{per}_{T}\left(\operatorname{tran}_{T}^{\triangle_{1}}\left(\triangle_{\epsilon \eta_{1}}\right) \cup M\right)=\operatorname{per}_{T}\left(\triangle_{\epsilon \eta_{1}}\right) \cup \operatorname{per}_{T}(M)
$$

is finite. By (3.17), it follows that $\operatorname{per}_{T}(\{z \in Q$, $\operatorname{dist}(z, \partial Q) \geq \epsilon\})$ is finite. This implies inequality (2.11) and concludes the proof of Theorem 2.4(ii). 
3.5.3. Proof of Theorem 2.4(iii). The proof is analogous to the proof of inequality (3.4).

Suppose, on the contrary, that

$$
\liminf _{\epsilon \rightarrow 0^{+}}\left\{\operatorname{per}_{T}(z) \mid \operatorname{dist}(z, \partial Q)<\epsilon\right\}=M<\infty .
$$

Then there is a $\left\{n_{k}\right\} \subset Q, n_{k} \rightarrow \partial Q$ such that $\operatorname{per}_{T}\left(n_{k}\right)=M$.

Since the pentagon $Q$ splits into a disjoint union of periodic cells located at a positive distance from $\partial Q$ and the transient orbit $\operatorname{tran}_{T}^{\triangle_{1}} \triangle_{1}$ (Lemma 3.11), we may assume that $\left\{n_{k}\right\} \subset \operatorname{tran}_{T}^{\triangle_{1}} \triangle_{1}$. This means that there is a sequence

$$
\left\{m_{k}\right\} \subset \triangle_{1}
$$

of preimages of $\left\{n_{k}\right\}$ such that

$$
\operatorname{per}_{F}\left(m_{k}\right) \leq \operatorname{per}_{T}\left(n_{k}\right)=M \text {. }
$$

Moreover, since $n_{k} \rightarrow \partial Q$, by Lemma 3.12 (the second part of the inequality (3.16)) we obtain

$$
m_{k} \rightarrow[d, q] \text { as } k \rightarrow \infty .
$$

From (3.19), (3.20), (3.21) we obtain

$$
\liminf _{\epsilon \rightarrow 0^{+}}\left\{\operatorname{per}_{F}(z) \mid \operatorname{dist}(z,[d, p])<\epsilon\right\} \leq M .
$$

Inequality (3.22) contradicts key Lemma 3.4(ii).

This concludes the proof of (iii) and it also concludes the proof of Theorem 2.4.

\section{Discussion}

Invariant curves and a more general setup. For the map $T$ considered we show that there is a natural decomposition of $\mathbb{C}$ into two disjoint sets; in the interior of $Q$ almost all initial conditions are periodic (but the period is unbounded) and all points are periodically coded. In the complement $\mathbb{C} \backslash Q$ there are five wedges which are filled with periodic points and the remainder is aperiodically coded. As such the map possesses an invariant Jordan curve (the boundary of $Q$ ) on which the dynamics is aperiodic, and that has a finite number of 'corners'. The region of aperiodically coded points can trivially be seen to be non-transitive and nonergodic due to the foliation by IETs. The map also gives, in our opinion, the simplest example with unbounded return times that is not a product of interval exchanges.

Figure 7 shows some example trajectories for a map $T_{\alpha}$ that permits variation of the parameter $\rho$ to include irrational rotations. This map is defined on the atoms

$$
\begin{aligned}
& P_{0}=\left\{r e^{i s} \mid r>0, s \in(0, \pi)\right\}, \\
& P_{1}=\left\{r e^{i s} \mid r>0, s \in\left(\pi, \theta_{1}\right)\right\}, \\
& P_{2}=\left\{r e^{i s} \mid r>0, s \in\left(\theta_{1}, \theta_{2}\right)\right\}, \\
& P_{3}=\left\{r e^{i s} \mid r>0, s \in\left(\theta_{2}, 2 \pi\right)\right\}
\end{aligned}
$$




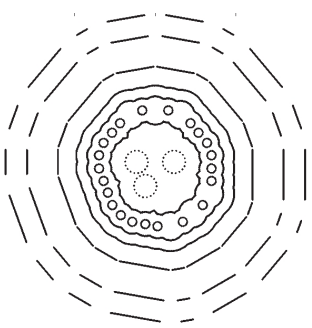

(a)

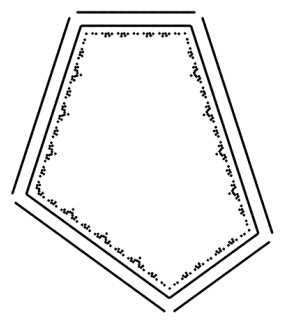

(b)

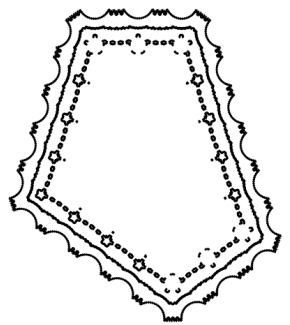

(c)

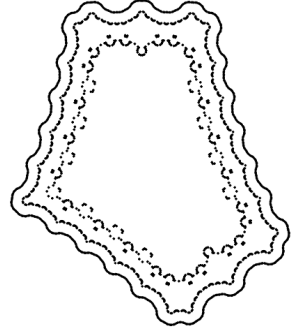

(d)

Figure 7. (a) Shows trajectories for ten different initial conditions with a family of invariant 36-gons for $T_{\theta}$ in the case $\theta=\frac{11 \pi}{9}=3.83972$. Trajectories for $T=T_{\theta}$ are shown in (b) $\theta=6 \pi / 5=3.76991$, (b) $\theta=3.77319$ and (c) $\theta=3.78319$. Each of (a)-(c) shows the computed trajectories starting at three different initial conditions, where the middle one is very close to the boundary of $Q$. In the perturbed cases (c) and (d) observe that some of the invariant lines in (b) break up into lines of periodic behaviour; others seem to persist.

by the rotations

$$
\begin{aligned}
& R_{0}(z)=e^{i \alpha} z-1+\rho, \\
& R_{1}(z)=e^{3 i \alpha} z-1+\rho, \\
& R_{2}(z)=e^{4 i \alpha} z-1+\rho, \\
& R_{3}(z)=e^{2 i \alpha} z-1+\rho,
\end{aligned}
$$

where $\rho=e^{i \alpha / 6}$. The piecewise rotation $T_{\alpha}$ is defined as $T z=R_{j} z$ if $z \in P_{j}$ and we choose $\theta_{1}=-2 \alpha$ and $\theta_{2}=\pi-\alpha$ modulo $2 \pi$. This reduces to the case above if $\alpha=6 \pi / 5$, and the map is well defined for an interval of $\alpha$ such that the ordering $\pi<\theta_{1}<\theta_{2}<2 \pi$ (modulo $2 \pi$ ) is preserved.

In another paper [5] we examine maps similar to $T_{\alpha}$ and observe existence of a large number of invariant curves that are apparently nowhere smooth, similar to those observed in 22. It is a challenge for the future to understand whether these curves are numerical artifacts, whether they exist only for zero area sets of initial conditions (but nevertheless form boundaries to ergodicity of the exceptional set), or whether they are dynamical features that occupy positive area in the phase space.

\section{REFERENCES}

[1] R.L. Adler, B. Kitchens and C. Tresser, Dynamics of non-ergodic piecewise affine maps of the torus. Ergod. Th. \& Dynam. Sys. 21 (2001) 959-999. MR1849597 (2002f:37075)

[2] P. Ashwin, Non-smooth invariant circles in digital overflow oscillations. Proceedings of the 4th Int. Workshop on Nonlinear Dynamics of Electronic Systems, Sevilla (1996) 417-422.

[3] P. Ashwin and X.-C. Fu, Tangencies in invariant circle packings for certain planar piecewise isometries are rare. Dynamical Systems 16, 4 (2001) 333-345. MR1870524(2002k:37071)

[4] P. Ashwin and X.-C. Fu, On the geometry of orientation preserving planar piecewise isometries. J. Nonlinear Sci. 12 (2002) 207-240. MR.1905204 (2003e:37053) 
[5] P. Ashwin and A. Goetz, Invariant curves and explosions of periodic islands in systems of piecewise rotations. (In preparation, 2004.)

[6] Michael Boshernitzan, Rank two interval exchange transformations. Ergodic Theory and Dynamical Systems 8 (1988) 379-394. MR0961737 (90c:28024)

[7] J. Buzzi, Piecewise isometries have zero topological entropy. Ergod. Th. Dyn. Sys. 21 (2001) 1371-1377. MR 1855837(2002f:37029)

[8] A. Goetz, Perturbation of 8-attractors and births of satellite systems. Intl. J. Bifn. Chaos 8 (1998) 1937-1956. MR1670619 (2000b:37038)

[9] A. Goetz, Dynamics of a piecewise rotation. Continuous and Discrete Dynamical Systems 4 (1998) 593-608. MR.1641165 (2000f:37009)

[10] A. Goetz, A self-similar example of a piecewise isometric attractor. Dynamical systems (Luminy-Marseille, 1998), 248-258, World Sci. Publishing, River Edge, NJ (2000). MR.1796163 (2001k:37060)

[11] A. Goetz and Gauillaume Poggiaspalla, Rotations by $\pi / 7$. Nonlinearity 17(5) (2004) 17871802. MR.2086151

[12] E. Gutkin and H. Haydn, Topological entropy of generalized polygon exchanges. Bull. Amer. Math. Soc. 32 (1995) 50-57. MR1273398 (95c:58118)

[13] E. Gutkin and N. Simányi, Dual Billiards and necklace dynamics. Communications in Mathematical Physics 143 (1992) 431-449. MR.1145593 (92k:58139)

[14] B. Kahng, Dynamics of symplectic piecewise affine elliptic rotation maps on tori. Ergod. Th. E Dynam. Sys. 2 (2002) 483-505. MR1898801(2003d:37078)

[15] K.L. Kouptsov and J. H. Lowenstein and F. Vivaldi, Quadratic rational rotations of the torus and dual lattice maps. Nonlinearity 15 (2002) 1795-1842. MR 1938473

[16] Kapustov and Lowenstein and Vivaldi, Recursive tiling and geometry of piecewise rotations by $\pi / 7$. Nonlinearity 17 (2004) 371-395. MR2039048

[17] A. Katok and B. Hasselblat, Introduction to Modern Theory of Dynamical Systems, Cambridge University Press, Cambridge 1995. MR.1326374 (96c:58055)

[18] L. Kocarev, C.W. Wu and L.O. Chua, Complex behaviour in Digital filters with overflow nonlinearity: analytical results. IEEE Trans CAS-II 43 (1996) 234-246.

[19] J. H. Lowenstein and F. Vivaldi, Embedding dynamics for round-off errors near a periodic orbit. Chaos 10 (2000) 747-755. MR.1802663 (2001j:37074)

[20] A.J. Scott, C.A. Holmes and G.J. Milburn, Hamiltonian mappings and circle packing phase spaces. Physica D 155 (2001) 34-50. MR.1837203(2002e:37082)

[21] S. Tabachnikov, On the dual billiard problem. Adv. Math. 115 (1995) 221-249. MR.1354670 (96g:58154)

Department of Mathematical Sciences, University of Exeter, Exeter EX4 4QE, United Kingdom

Department of Mathematics, San Francisco State University, 1600 Holloway Avenue, San Francisco, California 94132 